\title{
Transport Properties of Multifilamentary Ag-Sheathed Bi-2223 Tapes under the Influence of Strain
}

\author{
Takanobu Kiss, Hans van Eck, Bennie ten Haken, and Herman H.J. ten Kate
}

\begin{abstract}
Current-voltage (I-V) characteristics in multifilamentary $\mathrm{Ag} / \mathrm{Bi2223}$ tapes are investigated as a function of mechanical strain. As is well known, the critical current, $I_{c}$, in axially elongated tape remains almost constant up to a strain around $0.5 \%$, then is followed by a sharp reduction. However, for larger elongations, a long tail in the $I_{\mathrm{c}}$-strain curve is observed, i.e., around $20 \%$ of the initial $I_{c}$ still remains even at $0.8 \%$ strain. The irreversible $I_{c}$ reduction indicates that the degradation comes from the break-down of superconducting filaments. However, it is observed that the rupture risk probability reduces as the strain is increased in the long tail. This anomaly suggests that the measured strain of the whole tape is not identical to that of the HTS filaments inside the tape. We propose a model to describe the mechanical properties of the tape. It is shown that 1) the break-down probability of the filaments is well described by the Weibull function if we calculate the influence of shearing between the superconducting filaments and the surrounding $A g$ sheath, 2) the $I_{\mathrm{c}}$-strain properties can be described accurately by the model, 3 ) transport $I-V$ characteristics can also be described simultaneously as a function of strain.
\end{abstract}

Index Terms-Bi-2223 tape, tensile strain, critical current, transport properties, high-temperature superconductors.

\section{INTRODUCTION}

$\mathrm{K}$ NOWLEDGE of the strain properties of superconducting tapes are essential for practical application. The typical mechanical feature of HTS multifilamentary tapes is that brittle ceramic filaments are surrounded by soft metal sheath such as $\mathrm{Ag}$ or Ag-alloy. Consequently, the mechanical response against strain is complicated, and then the strain dependence of the critical current, $I_{c}$, is very much different from that of low temperature superconductors (LTS). It was shown that $I_{\mathrm{c}}$ in conventional LTS tape was reduced reversible as strain, $\varepsilon$, was increased [1],[2]. The change of $I_{\mathfrak{c}}$ is thought to originate from the variation of the upper critical magnetic field, $B_{\mathrm{c} 2}$. This idea is supported by the scaling behavior of pinning force density under the influence of strain [1],[2]. In HTS tapes, however, it has almost always been

Manuscript received September 17, 2000. This work was supported in part by the NEDO, Japan, under Grant No. 99E12-005-1.

T. Kiss was with the Low Temperature Division, University of Twente, 7500 AE Enschede, The Netherlands, on leave from Kyushu University, Fukuoka 812-8581, Japan (e-mail: kiss@sc.kyushu-u.ac.jp, telephone: +8192-642-3910, fax: +81-92-642-3963).

Hans van Eck, Bennie ten Haken, and Herman H.J. ten Kate are with the Low Temperature Division, University of Twente, 7500 AE Enschede, The Netherlands. observed so far that 1) the reduction of $I_{\mathrm{c}}$ is irreversible and 2) the reduction ratio of $I_{\mathrm{c}}$ is independent of magnetic field, $B$. These results suggest that the $I_{c}$ reduction in HTS tapes mainly comes from the mechanical break-down of superconducting filaments in the tape [2].

The typical response for the axially elongated tape is as follows. The value of the initial $I_{\mathrm{c}}$ is hardly reduced up to a strain around $0.4 \%$. Then it drops sharply when the strain exceeds a critical value, $\varepsilon_{\mathrm{c}}$. It was reported [2] that the threshold is determined by the compressive strain in the HTS filaments remaining after the heat treatment during the fabrication process. When the strain is increased to be much larger than the critical value, the reduction of $I_{\mathrm{c}}$ shows a tendency to be saturated. In many cases, the value of $I_{c}$ still retains $20 \%$ of the initial value even when the strain is as large as $0.8 \%$.

However, the strain we can measure is the apparent value of the whole tape. It is hardly possible to measure the strain of each filament inside the tape directly. Taking into account the fact that the HTS tapes are composites consisting of elements having quite different mechanical properties, we can easily imagine that the apparent strain could be different from that of the HTS filaments. Nevertheless, only a few studies have been carried out to clarify the detailed mechanical properties in HTS tapes at the moment. Moreover, in spite of the significant number of studies on the strain effect, most of them are concentrated on the influence on $I_{c}$, whereas the performance of the HTS-based devices depends strongly on the sharpness of the transition in transport electric field $(E)$-vs.-current density $(J)$ curves. It is necessary to investigate the influence on transport $E$ - $J$ curves as well.

In this study, we investigate the influence of tensile stress on the transport $E-J$ characteristics in $\mathrm{Bi}-2223$ multifilamentary tapes over a wide range of strain. We propose a model to describe the mechanical properties as well as transport properties in the tape. The comparison between the model and the measured results will be discussed.

\section{EXPERIMENT}

A schematic diagram of the instruments for the measurements is shown in Fig. 1. The Bi-2223 tape sample was soldered on the U-shaped spring stage made of brass. The strain is applied by elongating the spring in the axial direction. The amount of the strain is monitored by strain gauges mounted on the stage [2]. In our experiment we defined the strain value from as-cooled condition in liquid nitrogen. 
Namely, we ignored the off-set strain value originated from cooling. Recently, Kitaguchi et al. have reported that about $0.2 \%$ compressive strain will be introduced in the tape at liquid nitrogen temperature because of the difference of thermal contracts between Ag sheathed HTS tape and brass stage [4]. If we take into account this off-set value, the strain value will be reduced about $0.2 \%$. Applying the tensile strain systematically, we measured extended $E-J$ curves of the tape by the four probe method. The distance between the voltage taps was $1 \mathrm{~cm}$.

\section{RESULTS AND DISCUSSION}

\section{A. $I_{c}$-strain properties}

In Fig. 2, we show the strain dependence of $I_{c}$, which is determined by the $1 \mu \mathrm{V} / \mathrm{cm}$ criterion. The value of $I_{\mathrm{c}}$ was almost constant up to the strain value of $0.5 \%$, then followed by a sharp reduction. However, in the large strain region we can see a long tail. Namely, the $I_{c}$ is still $20 \%$ of the initial value even when the strain is as large as $0.8 \%$. The reduction of $I_{\mathrm{c}}$ did not recover if we reduced the strain. Also, the strain dependence did not depend on magnetic field. These results suggest that the $I_{\mathfrak{c}}$ degradation originates from the mechanical break-down of the filaments. Therefore, it would be reasonable to assume that the reduction ratio of $I_{c}$ is approximately proportional to the rupture probability, $G$, of the filaments. That is, $G(\varepsilon)=I_{c}(\varepsilon) / I_{c}(0)$.

We can obtain the rupture risk probability as a function of $\varepsilon$ from the result of Fig. 2, i.e., $(d G / d \varepsilon) /(1-G)$. The result is shown in Fig. 3. Note that the rupture risk probability for a small increment of $\varepsilon$ increases rapidly at the threshold, however, it is reduced again as $\varepsilon$ further increases. Namely, in the long tail region, the rupture risk probability becomes smaller and smaller as the strain is increased. This anomaly strongly suggests that the measured $\varepsilon$ is not identical to the actual strain in the filament because of a shearing between the remained filaments and $\mathrm{Ag}$ sheath as the break-down occurs in some part of the filaments.

\section{B. Modeling}

In this study, we elongated the sample by expanding the spring stage in the axial direction. We modeled, therefore, the mechanical response of the tape by taking into account the one-dimensional axial elongation by a spring network model as shown in Fig. 4. For simplicity, only the right half side of the stage is shown. The network consists of two elements, i.e., the coil spring, which represents the elastic interaction of the HTS filaments, and a flat spring, which represents shearing between the filaments and the sheath. The spring constants of those two springs are $k_{1}$ and $k_{2}$ per unit length, respectively. It can be seen that the strain in the filament, $\varepsilon_{1}$, is smaller than the applied strain, $\varepsilon_{\mathrm{eff}}$, due to the shearing, $\varepsilon_{2}$. Moreover, the rupture probability of the filament, $G$, is assumed to be given by the well-known Weibull function. When the strain, $\varepsilon$, is smaller than the critical value, $\varepsilon_{c}$, prestrain in the filaments, due to thermal contraction, is released. Therefore, $G$ is

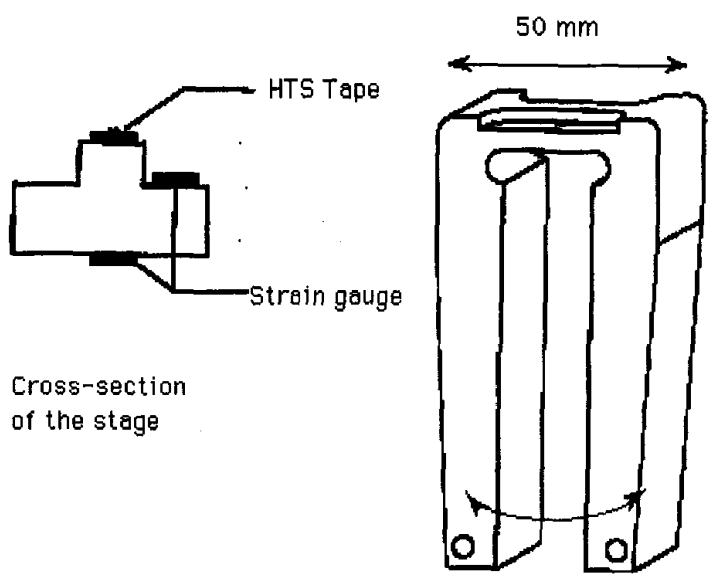

Fig. 1. Schematic diagram of the equipment which was used to apply strain to the HTS tape [2].

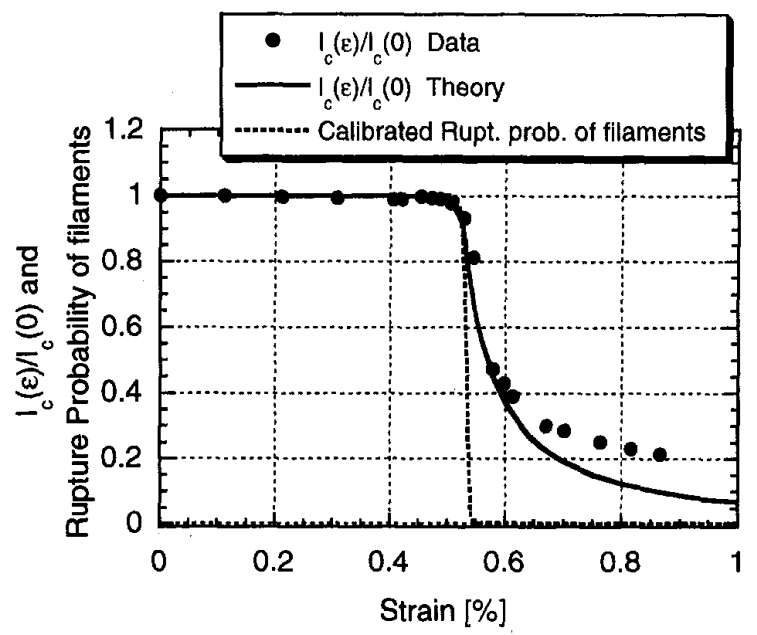

Fig. 2. $I_{\mathrm{c}}$-vs,-apparent strain. The initial value of $I_{\mathrm{c}}$ was $30 \mathrm{~A}$. The dots are measured results whereas the solid line is a calculation based on our model. The dotted line indicates the rupture probability of the filaments described by the Weibull function, (1.a) and (1.b), where the strain is calculated in the filaments. The numerical parameters are as follows: $\varepsilon_{c}=0.39, \varepsilon_{0}=0.1, \hat{m}=20$ and $k=0.17$.

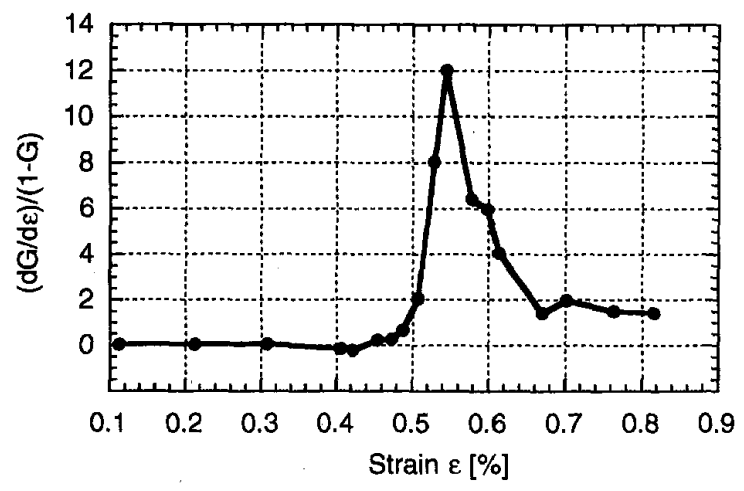

Fig. 3. The anomaly of the rupture risk probability, $(\mathrm{d} G / \mathrm{d} \varepsilon) /(1-G)$, obtained from the measured $I_{\mathrm{c}}-\varepsilon$ properties shown in Fig. 2 where $G(\varepsilon)=I_{\mathrm{c}}(\varepsilon) / \mathrm{I}_{\mathrm{c}}(0)$. 
assumed to be given by the following equation:

$$
\begin{aligned}
G(\varepsilon) & =1-\exp \left[-\left(\frac{\varepsilon-\varepsilon_{c}}{\varepsilon_{0}}\right)^{\hat{m}}\right] & \text { for } \varepsilon \geq \varepsilon_{c} \\
& =0 & \text { for } 0 \leq \varepsilon<\varepsilon_{c}
\end{aligned}
$$

where $\varepsilon_{0}$ is a scale parameter and $\hat{m}$ is a numerical constant describing the mode of destruction. The influence of the filament destruction in some parts of the cross-section will be reflected effectively as a variation of the shearing constant in the one-dimensional model. As the filament destruction progresses, the stress force should be sustained by the remaining filaments and sheath. Therefore, the effective shearing constant becomes smaller as the rupture probability increases. We assume that the ratio between $k_{1}$ and $k_{2}$ is a function of $\varepsilon$, then it is given by the following equation:

$$
\frac{k_{1}(\varepsilon)}{k_{2}(\varepsilon)}=k \frac{G(\varepsilon)}{1-G(\varepsilon)}
$$

where $k$ is a parameter characterizing the mechanical coupling strength between the filaments and the sheath. It can be expected that the value of $k$ will be small when the tape has a dense structure.

In order to clarify the relationship between the apparent strain and the real strain in the filaments, we analyze the properties of the model shown in Fig. 4. This problem can be transformed to make clear the relationship between the effective spring constant of the network and $k_{1}$. Because the relationship between stress and strain is the same as between voltage and current in a conductor, we can replace the mechanical model with the distributed resistance network model in an analogous way as shown in Fig. 5. $\rho_{1}$ is the resistance per unit length, which corresponds to $k_{1}$, whereas $1 / \rho_{2}$ is the leakage conductance, which corresponds to $k_{2}$. Consequently, we will obtain the relationship between $\varepsilon_{1}$ and $\varepsilon_{\text {eff }}$ if we obtain the relationship between effective resistance, $\rho_{\text {eff }}$, of the resistance network and $\rho_{l}$. Namely,

$$
\frac{\varepsilon_{1}}{\varepsilon_{\text {eff }}}=\frac{k_{\text {eff }}}{k_{1}}=\frac{\rho_{\text {eff }}}{\rho_{1}}
$$

Voltage, $V$, and current, $I$, in the distributed circuit are given by the following differential equations:

$$
\begin{aligned}
& d V=-I \rho_{1} d x, \\
& d I=-\frac{1}{\rho_{2}} V d x,
\end{aligned}
$$

then

$$
\frac{d^{2} V}{d x^{2}}=\frac{\rho_{1}}{\rho_{2}} V
$$

where $x$ indicates the distance along the tape. Solving the differential equation (4.c) with the boundary conditions:

$$
\begin{array}{ll}
V=V_{i} \text { and } I=I_{i}, & \text { for } x=0 \\
V=0, & \text { for } x=1
\end{array}
$$

we obtain the effective resistance, $\rho_{\text {eff }}$, for the unit length as

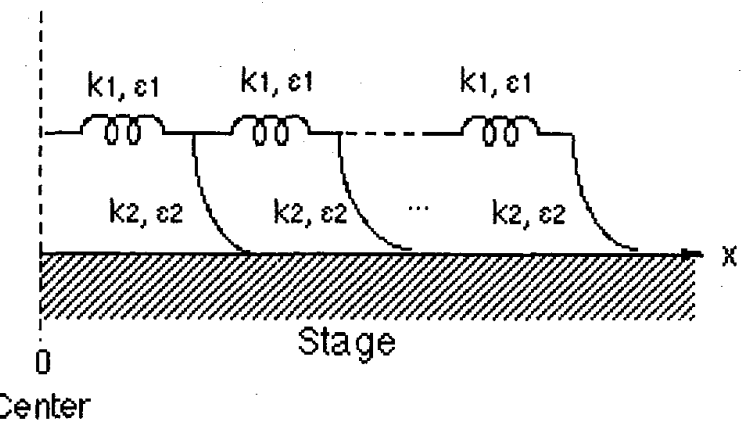

Fig. 4. Spring network model that takes into account the shearing between the HTS filaments and outer sheath.

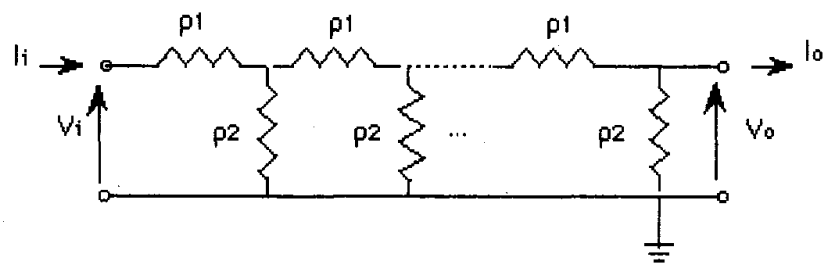

Fig. 5. Equivalent electric circuit model.

$$
\rho_{e f f} \equiv \frac{V_{i}}{I_{i}}=\sqrt{\rho_{1} \rho_{2}} \tanh \left(\sqrt{\frac{\rho_{1}}{\rho_{2}}}\right) .
$$

Therefore, the real strain in the filaments, $\varepsilon_{1}$, can be obtained from the measured strain, $\varepsilon_{\mathrm{eff}}$, by the following relationship:

$$
\varepsilon_{\text {eff }}=\varepsilon_{1} \sqrt{\frac{k G\left(\varepsilon_{1}\right)}{1-G\left(\varepsilon_{1}\right)}} \operatorname{coth}\left(\sqrt{\frac{k G\left(\varepsilon_{1}\right)}{1-G\left(\varepsilon_{1}\right)}}\right) .
$$

\section{Transport E-J curves under the influence of tensile strain}

In this section, we calculate the transport $E-J$ characteristics by expanding the mechanical model described in the preceding section. We confirmed that the $I_{c}$ reduction ratio by the strain does not depend on magnetic field. Therefore, we assume that the pining properties are not varied by the strain in the present experiment. Namely, the variation of $I_{c}$ comes only from the change of geometrical cross section area of the tape due to cracking.

The region where the filaments are destroyed reduces the effective cross-section area as schematically shown in Fig. 6. Due to the reduction of cross-section area, the current density in the remaining filaments is increased inversely proportional to $1-G$. As a result, the filament resistance in the crack region, $\rho_{c}$, is enhanced locally, and some part of the current will leak to the surrounding sheath. The relationship between the effective resistance and the filament resistance has already been given by (6) as described in the preceding section. Moreover, the length of the destroyed region per unit length will be given by $\varepsilon_{\mathrm{eff}}-\varepsilon_{1}$. Therefore, the total resistance per unit length will be given by a series connection of undestroyed region and the destroyed region.

$$
\rho_{\text {eff }}=\sqrt{\rho_{1} \rho_{2}} \tanh \left(\sqrt{\frac{\rho_{1}}{\rho_{2}}}\right)+\sqrt{\rho_{c} \rho_{2}} \tanh \left(\left(\varepsilon_{e f f}-\varepsilon_{1}\right) \sqrt{\frac{\rho_{c}}{\rho_{2}}}\right),
$$


where $\rho_{1}=\frac{E(I /(a S))}{I}$ and $\rho_{c}=\frac{E(I /[a S(1-G)])}{I}$,

$S\left(=8.5 \times 10^{-7} \mathrm{~m}^{2}\right)$ is the cross-section area of the tape, $a(=0.3)$ is the filling factor of the HTS filaments, and $\rho_{2}$ is the leakage resistance to the $\mathrm{Ag}$ sheath per unit length. Note that we assume that the essential $E(J)$ characteristics in the remaining filaments are the same as the initial characteristics.

We estimated the value of $\rho_{2}$ based on the following consideration. In the case when the resistivity in the HTS filament is equal to that of the sheath, the apparent resistivity estimated by (6) is also equal to that of the sheath. Using this condition, we evaluate the value of $\rho_{2}$. That is

$$
\rho_{2} \cong \frac{a}{S} \rho_{A g},
$$

where $\rho_{\mathrm{Ag}}$ is the resistivity in the sheath. We obtained the value $\rho_{\mathrm{Ag}}=3.3 \times 10^{-7} \Omega \mathrm{cm}$ at $77.3 \mathrm{~K}$ by measuring the linear resistance of the tape after breaking down the HTS filament mechanically. Using this value, we can estimate $\rho_{2}$ as 10 $\mu \Omega / \mathrm{cm}$ approximately in the present sample.

We have already proposed a method to describe transport $E-J$ characteristics in HTS based on a percolation model [3]

$$
\begin{array}{rlrl}
E(J) & =\frac{\rho_{F F}}{m+1} J\left(\frac{J}{J_{0}}\right)^{m}\left\{1-\frac{J_{c m}}{J}\right\}^{m+1}, & \text { for } & B \leq B_{G L}, J \geq J_{c m} \\
& =\frac{\rho_{F F}}{m+1}\left|J_{c m}\right|\left(\frac{\left|J_{c m}\right|}{J_{0}}\right)^{m}\{(10 . a)
\end{array}
$$

where $J_{\mathrm{cm}}$ is the minimum value of the critical current density in the statistic $J_{\mathrm{c}}$ distribution, $J_{0}$ is the scale parameter indicating the width of the $J_{c}$ distribution, $m$ is a parameter determining the shape of the $J_{\mathrm{c}}$ distribution, $\rho_{\mathrm{FF}}$ is the flux flow resistivity in uniform flux flow, and $B_{\mathrm{GL}}$ is the transition field where $J_{\mathrm{cm}}=0$ [3]. The initial $E-J$ characteristic in the present experiment can be described by this model well with the numerical parameters: $J_{\mathrm{cm}}=9.48 \times 10^{7} \mathrm{~A} / \mathrm{m}^{2}, J_{0}=2.80 \times 10^{8}$ $\mathrm{A} / \mathrm{m}^{2}, m=3.5$ and $\rho_{\mathrm{FF}}=10 \mu \Omega \mathrm{cm}$. Then, we finally obtain the strain dependence of the $E-J$ characteristics.

Comparison between the theory and the measured results is shown in Fig. 7. It can be seen that the model describes precisely the experimental results except in the very large strain region. The calculation results describe the detailed features of the experimental results: 1) before the sharp reduction of $I_{c}$, the degradation occurs first in the lower electric field region, 2) the variation of $n$ value can be described as well. The reduction of $I_{c}$, determined using 1 $\mu \mathrm{V} / \mathrm{cm}$ criterion, is also shown by the solid line in Fig. 2 . In the same figure, we plot the rupture probability of the filaments calculated by (1.a), (1.b) and (7) with the numerical parameters: $\varepsilon_{\mathrm{c}}=0.39, \varepsilon_{0}=0.1, \hat{m}=20$, and $k=0.17$. It can be seen that if we consider the influence of the shearing, the filaments break sharply when the applied strain exceeds the critical value. This is quite reasonable if we take into account

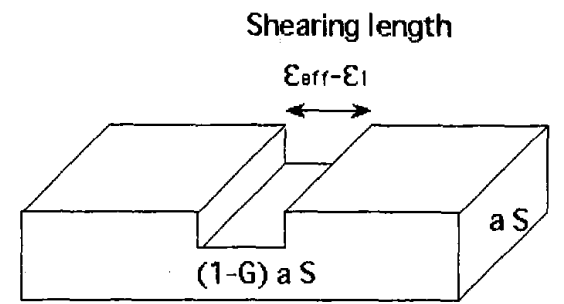

Fig. 6. In the ruptured region, the effective cross-section area is reduced inversely proportional to $1-G$. The length of the narrowed region is assumed to be equal to the shearing length.

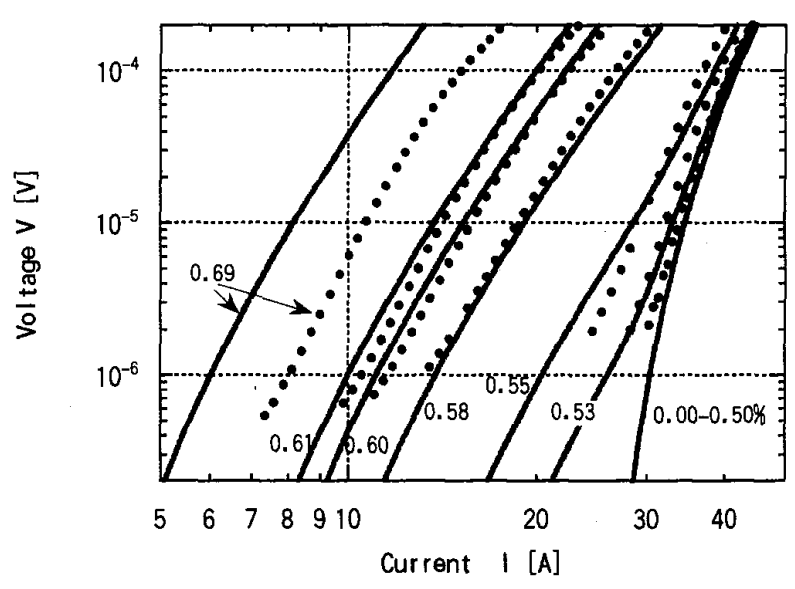

Fig. 7. Transport $E-J$ characteristics as a function of strain. The dots are measured results while the solid lines are the results obtained by the present model. The numbers beside the lines indicate the value of tensile strain.

the brittle properties of HTS filaments.

These results indicate that the proposed model can describe the mechanical properties as well as the transport current properties of the HTS tape.

\section{CONClusion}

We proposed a model to describe the mechanical properties of multifilamentary HTS tape. It is shown that 1) the breakdown probability of the filaments is well described by the Weibull function if we consider the influence of shearing, 2) the $I_{\mathrm{c}}$-strain properties can be described accurately by the model, 3) transport $I-V$ characteristics can also be described simultaneously as a function of strain.

\section{REFERENCES}

[1] J.W. Ekin, "Strain scaling law for flux pinning in practical superconductors, Part 1: Basic relationship and application to $\mathrm{Nb}_{3} \mathrm{Sn}$ conductors," Cryogenics, vol. 20, pp. 611-624, Nov. 1980.

[2] B. Ten Haken, "Strain effects on the critical properties of high-field superconductors," Ph.D. Thesis, University of Twente, 1994.

[3] T. Kiss, T. Matsushita, and F. Irie, "Relationship among flux depinning, irreversibility and phase transition in a disordered HTS material," Supercond. Sci. and Technol, vol. 12, pp. 1079-1082, Dec. 1999.

[4] H. Kitaguchi, K. Itoh, K. Kumakura, T. Takeuchi, K. Togano, and H. Wada, "Strain effect in Bi-based oxyde/Ag superconducting tapes," IEEE Trans. Appl. Supercond. 2000, submitted for publication. 